\title{
Radiation Damage Effects in the LHCb Vertex Locator (VELO)
}

\author{
Henry Brown*t \\ University of Liverpool \\ E-mail: henry.brownecern.ch
}

The VErtex LOcator (VELO) is a detector at the LHCb experiment. It is a silicon microstrip detector, and surrounds the beam collision region at LHCb. Its main purpose is to reconstruct and trigger on primary and secondary vertices with high precision. It is active in a high radiation environment, and requires radiation damage monitoring. The first results of the radiation damage monitoring studies performed within the VELO group are described here, using a variety of scans, analyzing the changes in Charge Collection Efficiency (CCE), currents, and depletion voltage. This paper describes monitoring of data accumulated up to $433 p b^{-1}$.

R\&D 11, 10th Workshop

6-8th July 2011

Firenze, Italy

\footnotetext{
*Speaker.

$\dagger$ On behalf of the LHCb VELO group
} 


\section{Introduction}

LHCb is a precision flavour physics experiment at the Large Hadron Collider. It is primarily designed for the detection of $\mathrm{b}$ hadrons for the study of $\mathrm{CP}$ violation, and the search for new physics in rare decays. The VErtex LOcator is the vertex detector at $\mathrm{LHCb}$, providing high precision separation of primary and secondary vertices. The VELO sensors are positioned only $8 \mathrm{~mm}$ away from the beamline during data taking, and as such are exposed to a very high radiation dose. The estimated maximum fluence for a single sensor is approximately $0.7 \times 10^{14} n_{e q} \mathrm{~cm}^{-2}$ per $1 \mathrm{fb}^{-1}$ [1] at the tip of the sensor. This is elaborated in further detail in Figure 2. This results in a high radiation damage environment for the VELO and the detector is monitored for the effects of the radiation damage.

\section{The LHCb VErtex LOcator}

The VELO consists of 84 approximately semi-circular sensors, of two types (sensors giving radial information and angular information, called $\mathrm{R}$ and $\phi$ ). R and $\phi$ sensors are placed back-toback to give 42 individual modules. The sensors are arranged in two detector halves, known as the $\mathrm{A}$ and $\mathrm{C}$ side. A view of one half has been included in Figure 1. The nominal operating temperature of the sensors is $-10^{\circ} \mathrm{C}[2]$. The radiation damage monitoring scans documented here all require special types of data taking.

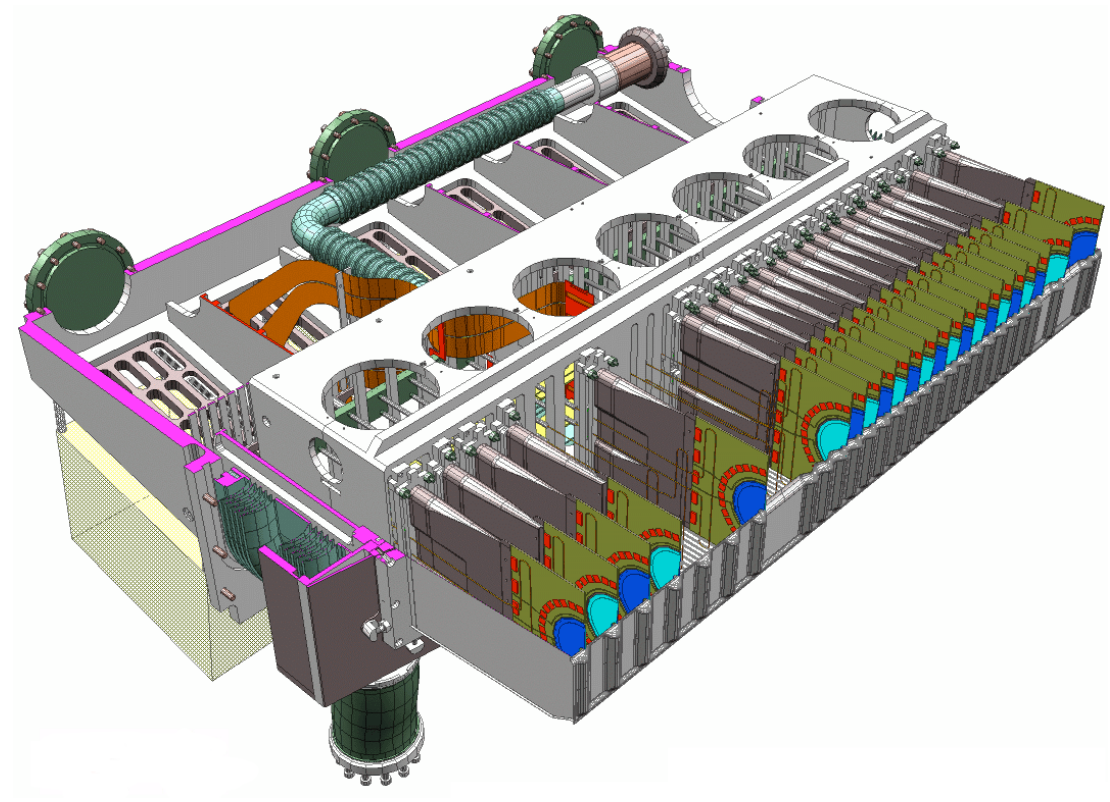

Figure 1: A graphic displaying one half of the VELO. The corrogated RF foil can be seen in the forefront of the picture, covering the 21 detector stations. The modules are mounted onto bases for support and cooling purposes, which in turn connect to the bi-phase $\mathrm{CO}_{2}$ cooling system.

The majority of the sensors are n+-on-n design, using strongly doped n-type implants in n-type bulk silicon. In addition, there are two n+-on-p design sensors with strongly doped n-type implants 
in p-type bulk silicon. The first results on their performance with respect to the radiation damage accumulated up until July 2011 are presented below. The expected radiation damage varies strongly with radius across an individual sensor. In addition, there is also a dependence on the position along the z-axis, which translates to the 'station number'. This is demonstrated in Figure 2. The geometry of LHCb is documented in the technical design report [3].

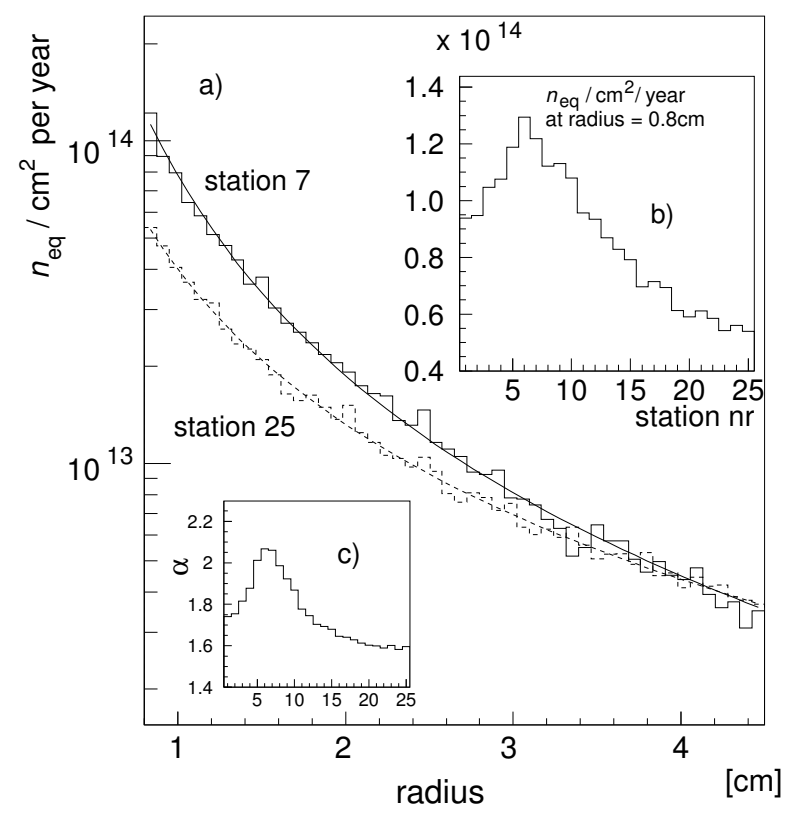

Figure 2: The expected radiation damage profile for the VELO sensors. The profile is modelled using $\phi=N r^{-\alpha}$ where the $\mathrm{N}$ (fluence for $R=0.8 \mathrm{~cm}$ ) and $\alpha$ (radial dependence) are presented in the inset graphs above, as well as two sample whole-sensor profiles. Main figure (a) presents two radial profiles for different stations at two different positions from the interaction point, whilst sub-figure (b) describes the maximum fluence $(\mathrm{N})$ per station and sub-figure (c) describes the radial dependence (alpha) per station [1].

The range of studies performed to monitor and understand the radiation damage are discussed below.

\section{IV Scans}

The IV scan monitoring uses the high voltage system for the reverse biasing of the silicon strips. The scan involves a ramp from $0 V$ to the operating voltage (for the data presented, this is $150 \mathrm{~V}$ ) and back down in $10 \mathrm{~V}$ steps, at each point recording the current. These scans take approximately 20 minutes, and can be performed between LHC fills. They are taken approximately weekly, though they may be taken as required, and after any period of annealing has occured such as after a power cut, which can have affects upon the cooling system. This scan can in the future be used to extract information on the fluence received by the detectors.

Figure 3 shows the change in current in one sensor during the period of the LHC physics run, in which some interesting features can be observed. During data taking 2010, a linear increase 


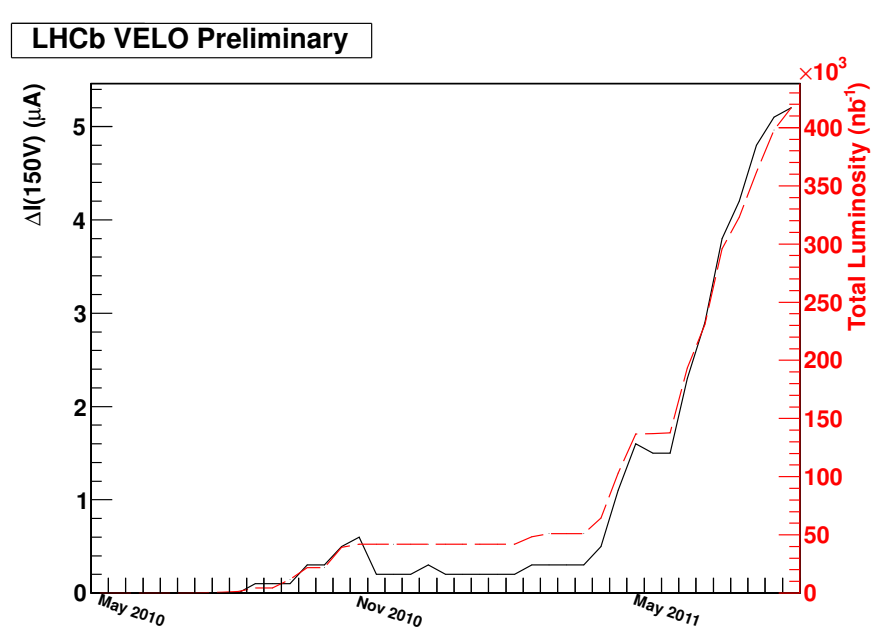

Figure 3: The solid line shows the change in current for one particular n+-in-n type sensor between the start of the first LHC physics run in May 2010 and July 2011. The sensor was operated at $150 \mathrm{~V}$ bias. The dashed superimposed line is the total integrated luminosity delivered by the LHC to LHCb.

in current can be observed with respect to the integrated delivered luminosity. This is followed by a significant drop in current, due to the VELO being annealed over the LHC shutdown period (of which approximately 50 days were spent at room temperature). The average change in current across the normal (e.g. ones that did not have high current at production) $n$-in-n sensors during the period from May 2010 to July 2011 is $\Delta I=2.2 \mu A$ per $100 p b^{-1}$. The corresponding number for the p-type sensors in the VELO is $\Delta I=2.5 \mu A$ per $100 p b^{-1}$. Further to the two cases described above, there is a third group of sensors in the VELO, corresponding to sensors that drew unusually high current at production, potentially arising from defects internal on the silicon surface. These have reduced their current with increased radiation damage, they have now reached a stable state. This is clearly seen in Figure 4. 


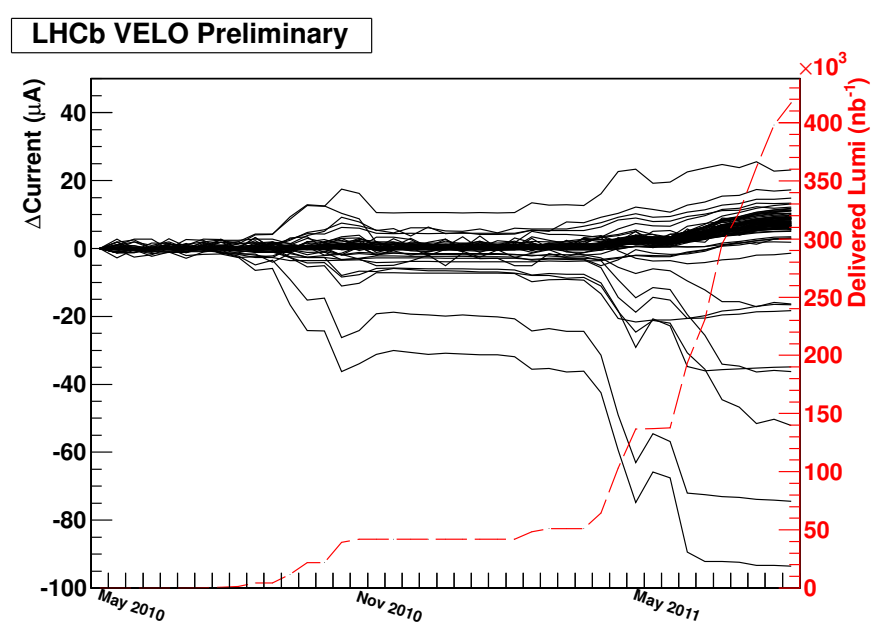

Figure 4: The solid lines shows the change in current for all the sensors in the VELO.

\section{Noise vs Voltage Scan}

The second technique used to monitor the radiation damage is the noise vs voltage scan. Again this data is taken during periods between LHC runs, and takes between 20 minutes and an hour to collect, depending on the granularity. An automated stepping procedure is used with data acquired at voltage steps from $0 \mathrm{~V}$ to $150 \mathrm{~V}$. The analysis concept is illustrated in Figure 5. The noise is expected to minimise once the detector reaches its full depletion voltage. Hence the voltage at which 1/Noise approaches its maximum is extracted and labelled the 'Effective Depletion Voltage' (EDV). Tracking how the EDV changes over time allows the monitoring of the radiation damage. This technique is useful as the scans can be performed quickly and do not interrupt physics operations, unlike the charge collection efficiency CCE scan which is discussed below.

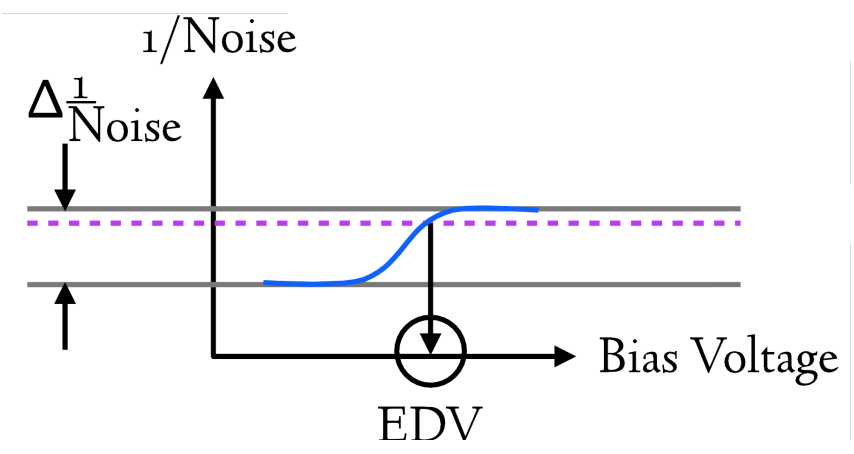

Figure 5: The technique used to determine the effective depletion voltage in the noise vs voltage scan. $1 /$ Noise is plotted as a function of the bias voltage. The EDV is defined as when the $\Delta \frac{1}{\text { Noise }}$ is $90 \%$ of the maximum value.

In the analysis, each $\mathrm{R}$ sensor is divided into four radial zones. From the expected radiation damage profiles of the sensors, the innermost zones should have experienced the highest change 
in depletion voltage. For an n-in-n sensor the depletion voltage is expected to initially drop until the sensor undergoes type inversion. Figure 6 indeed shows that the EDV of the innermost zone of the sensors have changed most, and the outermost zone have changed least, in agreement with the expectation. The extracted EDV results are normalized to the value measured at the start of the LHC physics operation in 2011, the ratio (as used in the results in Figure 6) is defined as: $\Delta E D V=\frac{E D V_{\text {measured }}}{E D V_{\text {production }}}$.

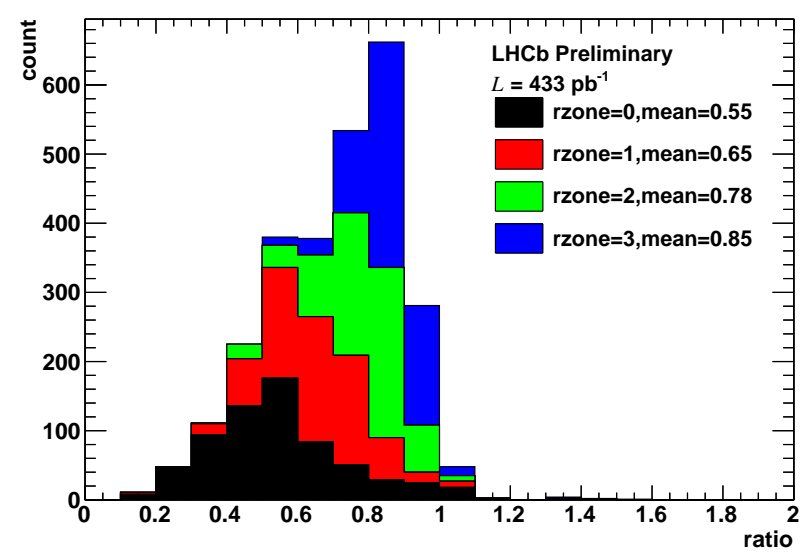

Figure 6: Results for the LHCb VELO noise scan method. Zone 0 corresponds to the innermost (closest to the beam) part of the sensor and zone 3 the outermost.

\section{Charge Collection Efficiency Scan}

The charge collection efficiency scan (or CCE scan) is one of the most effective tools available for monitoring radiation damage in silicon. In the VELO it requires the use of particle tracks (thus requiring collisions), implying the loss of this data for physics purposes. Hence, it is only performed approximately every 3-6 months. The CCE scan is performed as follows: Every fifth sensor is designated as a test sensor. The other four sensors, comprising two before each test sensor and two after are operated at the nominal $150 \mathrm{~V}$ bias and used for particle tracking. Data is taken with the test sensors operated at a range of voltages from $0 \mathrm{~V}$ to $150 \mathrm{~V}$. The fitted particle tracks are extrapolated to the test sensor and the charge on the strips around the intercept point of the track is determined. The data taking scans are repeated for all patterns, so that each sensor is tested. The voltage scans and change of pattern procedure are automated. The procedure takes around 2 hours to complete.

Figure 7 shows the ADC count distribution in one sensor at one voltage. The most probable value (MPV) of the Landau distribution is extracted. The MPV as a function of voltage is then obtained as shown in Figure 8. An effective depletion voltage is determined from the point where the MPV approaches its maximal value. The point at which $80 \%$ of the full depletion number of electrons is reached is defined as the effective depletion voltage. This was chosen as it gives the optimal agreement between the CCE scan taken at the start of the LHC physics run and the depletion voltages determined at production of the sensors. 


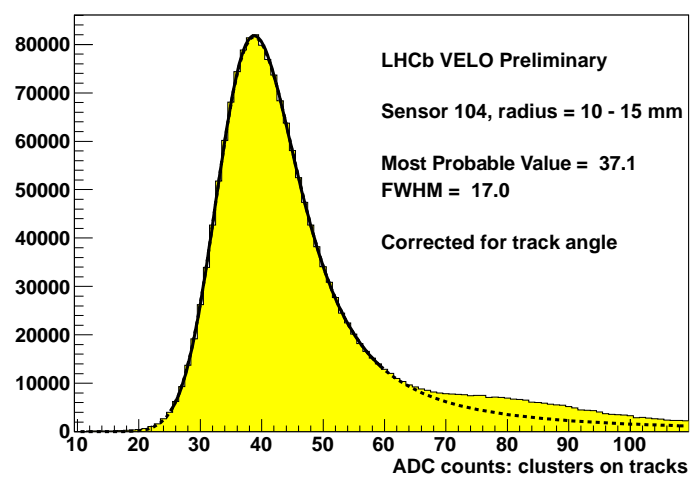

Figure 7: An example of a Landau for one particular sensor at one voltage.

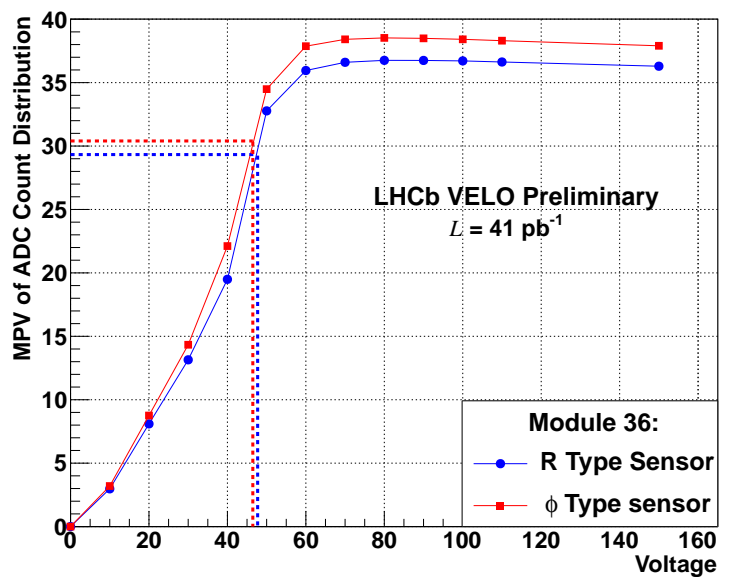

Figure 8: The MPV of the Landau distribution as a function of voltage for two sensors.

Similarly to the noise vs voltage scan, the sensors are divided into different bands of radius, from innermost to outermost bands. The amount of radiation damage should decrease with radius. The measured change in depletion voltage is greatest at the innermost section of the sensors, as shown in figure 9 after an integrated luminosity of $40 \mathrm{pb}^{-1}$. 


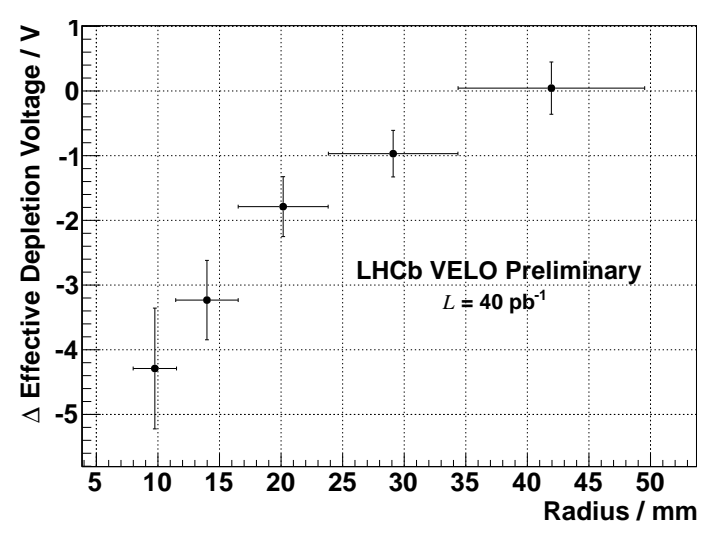

Figure 9: The average change in depletion voltage across all sensors plotted as a function of the radius.

\section{Conclusion and Summary}

The first effects of radiation damage have been observed in the LHCb VELO. Generally, the currents have been rising proportionally to the delivered luminosity. Some sensors with high initial currents have reduced their currents with radiation. A drop in the effective depletion voltage with radiation has been measured in the noise vs voltage and CCE scans. Type inversion in the $n+-i n-n$ sensors is expected to be observed soon (with respect to the data presented here), at this point the depletion voltage of the sensors will start to increase [4] [5] with respect to fluence. The detailed studies of the radiation damage will be used to determine the optimal operational voltage and periods of beneficial annealing.

The LHCb VELO provides an important laboratory for the silicon community to study radiation damage effects. The presence of both n-in-n and n-in-p sensors, the proximity to the LHC beam, and the wide variation of fluences across the sensors allows a unique range of comparative studies to be performed. The results will help determine the strategy for the longterm operation of the LHC silicon detectors and influence the designs for the future LHC upgrades.

\section{References}

[1] LHCb VELO Technical Design Report, CERN/LHCC 2001-011 (2001)

[2] A.A Alves et al., The LHCb Detector at the LHC, Jinst 3 (2008), S08005

[3] The LHCb Collaboration, LHCb Technical Design Report, Reoptimized Detector Design and Performance, CERN-LHCC-2003-064

[4] F. Lemeilleur, et al., Neutron-induced radiation damage in silicon detectors, IEEE TRANSACTIONS ON NUCLEAR SCIENCE, VOL. 39, NO. 4,1992 551

[5] F. Lemeilleur, et al., Neutron, Proton, and Gamma Iraddiations of Silicon Detectors, IEEE TRANSACTIONS ON NUCLEAR SCIENCE VOL. 41, NO. 3, JUNE 1994425 